Théologiques

Théologiques

\title{
Le visage du charisme
}

Une page de Proust

\section{Isabelle Kalinowski}

Volume 17, numéro 1, 2009

Les charismes

URI : https://id.erudit.org/iderudit/039497ar

DOI : https://doi.org/10.7202/039497ar

Aller au sommaire du numéro

\section{Éditeur(s)}

Faculté de théologie et de sciences des religions, Université de Montréal

ISSN

1188-7109 (imprimé)

1492-1413 (numérique)

Découvrir la revue

Citer cet article

Kalinowski, I. (2009). Le visage du charisme : une page de Proust. Théologiques, 17(1), 33-50. https://doi.org/10.7202/039497ar
Résumé de l'article

Cet article propose une lecture sociologique du passage de $\grave{A}$ la recherche $d u$ temps perdu où le jeune Marcel rencontre la duchesse de Guermantes et réaffirme, après un premier moment de "déception ", sa croyance dans le " charisme " de cette dame. Fondé sur la théorie du charisme de Max Weber, ce commentaire cherche à prolonger cette dernière en insistant sur deux dimensions mises au jour par Proust : la différence entre croyance

charismatique et croyance " traditionnelle ", d'une part, et, de l'autre, le rôle de la perception du corps dans la genèse de l'enchantement charismatique. d'utilisation que vous pouvez consulter en ligne.

https://apropos.erudit.org/fr/usagers/politique-dutilisation/ 


\title{
Le visage du charisme
}

\section{Une page de Proust}

\author{
Isabelle KALINOWSKI* \\ Sociologie \\ École Normale Supérieure, Paris
}

À la recherche $d u$ temps perdu peut aussi être lue comme une longue méditation sur la question du charisme. C'est du point de vue de l'adepte que Proust l'envisage. L'adepte croit au charisme de quelqu'un d'autre, il est le porteur de la croyance dans un charisme qui n'est pas le sien. Dans le récit proustien, le narrateur n'épouse pas le moment où cette croyance est encore pleine, ardente; elle est toujours vue depuis un après. Celui qui la décrit sait que la foi charismatique est mortelle, comme certaines amours. C'est un œil prévenu, arraché à l'adhésion première du fidèle, qui revient scruter le fascinant phénomène que constitue une foi si entière. La Recherche est un inventaire de ces ferveurs déçues, qui peuvent s'effondrer sans bruit le jour venu, se dissiper soudain en laissant, intacte, l'énigme de leur consistance passée: comment ont-elles pu se présenter sous un jour si compact, adopter une forme si absolue, alors qu'elles étaient susceptibles d'être perdues? Les expériences charismatiques dont le narrateur accumule les détails (les siennes et celles qu'il observe passionnément chez les autres) sont autant de spécimens d'une seule et même espèce dont il cherche inlassablement à cerner les contours. L'extrait qui a été sélectionné ici, une page de Combray où l'on voit Marcel rencontrer pour la première fois une dame qui l'avait longtemps fait rêver, la duchesse de Guermantes, n'est qu'un exemple parmi bien d'autres de l'attention portée par Proust à l'enchantement charismatique. Beaucoup de pages de la Recherche auraient pu être analysées de la même manière.

* Isabelle Kalinowski, chercheur au CNRS, travaille à Paris dans le laboratoire «Pays germaniques » de l'École Normale Supérieure. Elle est spécialiste de la sociologie religieuse de Max Weber, dont elle a édité et traduit, tout récemment, Le judaïsme antique (Flammarion, à paraître en janvier 2010). Elle prépare actuellement un livre intitulé Le charisme. Autour de Max Weber. 
Le choix de ce texte plutôt que d'un autre a d'abord été dicté par le souci de mettre en avant la différence entre expérience charismatique et expérience amoureuse: non seulement la première ne se confond pas avec la seconde, mais elle n'est même pas une variété particulière de celle-ci; au contraire, on pourrait penser que, dans la Recherche tout au moins, c'est l'amour qui appartient au genre plus général de l'expérience charismatique, dont il ne parvient jamais à se dégager vraiment et dont il ne constitue au fond qu'un sous-ensemble sans privilège spécifique. Le rapport d'allégeance qu'instaure la croyance dans le charisme d'un être n'a pas besoin d'être déclenché par une attirance amoureuse, même s'il peut se conjuguer avec celle-ci. Il active le niveau d'une attente à la fois extrêmement crue et extrêmement sublimée.

Le second motif du choix de cet extrait tient à la précision avec laquelle Proust aborde ici une dimension généralement ignorée par les sciences sociales, parce que les outils permettant de la saisir ne leur sont guère familiers: je veux parler de la perception corporelle, de tous les événements, infimes mais décisifs, qui, dans l'expérience charismatique, interviennent à même la peau, entre un regard et la surface d'un corps. Proust postule que c'est dans le regard sur un corps, dans la présence commune d'un corps regardant et d'un corps regardé, que le charisme, justement, "prend corps » et se met à exister pour celui qui est saisi par l'enchantement charismatique. Ce saisissement est d'abord une expérience physique, même s'il est préparé par toute une activité de l'esprit, du mythe et de l'imagination. Le texte sur la duchesse de Guermantes recense avec finesse la succession de ces différents moments, qui s'entrelacent mais restent organisés autour de l'instant de co-présence de deux corps: celui-ci est le pivot de toute l'expérience charismatique.

Balzac remarque, dans un texte de 1830 , que l'observation des physionomies humaines n'a d'abord été envisagée, par ceux qui ont tenté d'en faire une science, qu'en vue de l'examen de certains «monstres» sociaux:

La crânologie, la physiognomonie, n'ont été appliquées qu'aux illustres assassins, qu'aux célèbres imbéciles, qu'aux furieux érotiques. Les bosses de la tête, le feu des yeux, les battements du cœur ont été analysés; mais on a négligé la délicatesse du palais, la capacité et les mouvements de l'estomac. Tous les hommes ne sont pas assassins, imbéciles ou érotiques, mais tous les hommes ont une bouche, un estomac et un ventre. Cette vérité, si évidente, aurait dû déterminer MM. Lavater, Gall et Cie à abandonner les spécialités pour généraliser l'application de leur science. (Balzac 1978, 147-148) 
De fait, l'attention portée par certains écrivains à la description des plus remarquables qualités physiques de leurs personnages s'apparente à une forme de physiognomonie "généralisée », fondée sur le principe d'une corrélation directe entre les propriétés morales et sociales et l'apparence extérieure des individus. Mais le souci de scruter les visages et les corps n'a pas pour seule motivation le désir de percer l'identité des personnes par delà l'image qu'elles veulent donner d'elles-mêmes; il est également inspiré par un postulat qui, pour avoir été rarement énoncé comme tel, n'en détermine pas moins, aux yeux de certains écrivains, toute la dynamique des interactions humaines: l'idée que les échanges entre les individus passent par les corps avant de faire intervenir le niveau des intentions et des discours explicites. De ce point de vue, l'extrait de la Recherche choisi ici s'avère particulièrement significatif, puisqu'il dépeint une rencontre strictement visuelle et non verbale entre les deux protagonistes ${ }^{1}$. À l'occasion de la célébration d'une messe dans l'église de Combray, Marcel aperçoit la duchesse de Guermantes installée dans la chapelle latérale réservée à sa famille. Il n'engage pas de dialogue avec elle, et ne peut davantage se rapprocher de cette dame ni faire varier ses angles de vue en se déplaçant; il entre en contact avec elle par le seul regard, et par un regard fixe, orienté dans une perspective unique. Le texte décrit alors, par la retranscription fictive d'un "flux de conscience», la manière dont Marcel perçoit le visage et le corps de Mme de Guermantes. Le corps perçu n'est pas un simple support, une simple illustration de son identité. Il n'est pas la manifestation extérieure et seconde d'une qualité intrinsèque. Il est la duchesse toute entière, dont l'être, pour le jeune garçon, n'affleure jamais aussi explicitement que dans l'instant où il lui «saute aux yeux».

\section{La tradition et ses images}

Jamais dans la promenade du côté de Guermantes nous ne pûmes remonter jusqu'aux sources de la Vivonne, auxquelles j'avais souvent pensé et qui avaient pour moi une existence si abstraite, si idéale, que j'avais été aussi

1. L'échange verbal constitue évidemment aussi une forme de perception du corps, comme je l'ai souligné en étudiant ailleurs le rôle de la voix dans la production de la croyance charismatique. Mais la parole agit alors à un double niveau, la perception sensible de la voix et la compréhension intellectuelle du message énoncé, et il est difficile de mesurer la part qui revient à l'une et à l'autre dans la genèse du charisme. Le dispositif que l'on rencontre dans ce texte de Proust, un cas de perception exclusivement visuelle et muette, offre une situation expérimentale pour analyser la production du charisme indépendamment de toute émission discursive. 
surpris quand on m'avait dit qu'elles se trouvaient dans le département, à une certaine distance kilométrique de Combray, que le jour où j'avais appris qu'il y avait un autre point précis de la terre où s'ouvrait, dans l'Antiquité, l'entrée des Enfers. Jamais non plus nous ne pûmes pousser jusqu'au terme que j'eusse tant souhaité d'atteindre, jusqu'à Guermantes. Je savais que là résidaient des châtelains, le Duc et la Duchesse de Guermantes, je savais qu'ils étaient des personnages réels et actuellement existants, mais chaque fois que je pensais à eux, je me les représentais tantôt en tapisserie, comme était la Comtesse de Guermantes, dans le "Couronnement d'Esther » de notre église, tantôt de nuances changeantes comme était Gilbert le Mauvais dans le vitrail où il passait du vert chou au bleu prune selon que j'étais encore à prendre de l'eau bénite ou que j'arrivais à nos chaises, tantôt tout à fait impalpables comme l'image de Geneviève de Brabant, ancêtre de la famille de Guermantes, que la lanterne magique promenait sur les rideaux de ma chambre ou faisait monter au plafond - enfin toujours enveloppés du mystère des temps mérovingiens et baignant comme dans un coucher de soleil dans la lumière orangée qui émane de cette syllabe: «antes». (Proust 1987, 284)

Avant la rencontre proprement dite entre Marcel et madame de Guermantes, le préambule qu'on vient de lire replace la duchesse dans une série de figures mi-réelles, mi-fabuleuses, appartenant à l'univers de la légende. Ce passage est construit comme une avancée progressive dans le sens de la dématérialisation: le château des Guermantes, d'abord, est un lieu réel, mais déjà inaccessible; la tapisserie, en second lieu, fait entrer dans la sphère de la représentation, même si son étoffe est encore palpable; le vitrail, ensuite, est ancré dans la matérialité du verre mais déjà tout de lumière et de couleurs; puis vient l'image de Geneviève de Brabant, projetée au plafond par la lanterne magique, qui ne possède plus d'épaisseur tangible; enfin, l'éclat orangé éveillé par la syllabe "antes " ne peut même plus être vu, il est cette fois exclusivement imaginé. Ces étapes vers toujours plus d'immatérialité préparent, en le dramatisant par avance, le moment où le jeune Marcel sera confronté, dans sa réalité soudain concrète, à la femme dont le nom réveillait en lui ces visions de l'ordre du merveilleux.

Il serait inexact de regarder ce prélude comme une explication logique des causes de la croyance charismatique, et du saisissement que ressentira Marcel à la vue de la duchesse. Ce n'est pas, nous allons le voir, le prestige de la famille de Guermantes qui fonde le charisme de cette dame. De la même façon que la foi charismatique ne se confond pas avec le saisissement amoureux, même si elle peut se combiner avec ce dernier, elle ne procède pas non plus au premier chef (sans être pour autant en aucune manière 
incompatible avec lui) du type d'allégeance spécifique qui est dépeint dans ce passage sur la généalogie mythique des Guermantes. L'admiration vouée par le jeune homme à cette famille ne constitue pas de prime abord une forme de dévouement à son autorité charismatique mais à son autorité traditionnelle. La lignée des Guermantes représente, typiquement, une instance de la tradition, dont l'histoire, éminemment longue (les «mérovingiens»), est inscrite dans la pierre du château et dans celle de l'église. Les attaches parisiennes de la famille de Marcel comme celles des Guermantes reproduisent, sans la modifier, la structure fondamentale qui porte un jeune bourgeois de Combray à reconnaître l'autorité traditionnelle des châtelains du village.

Proust décrit ici le fonctionnement de la croyance traditionnelle avec beaucoup de justesse, en invitant à reconsidérer ce qui la lie à des représentations "routinisées » et ce que signifie, au fond, une telle "routinisation». Les images des Guermantes énumérées dans le texte sont bien des images «quotidianisées », usées parce qu'elles ont été vues mille fois, comme une distraction pendant la messe du dimanche ou dans l'assoupissement du début de la nuit, sous les rais colorés de la lanterne magique; mille fois encore, quand l'enfant, à Combray, s'est promené "du côté de Guermantes ». Mais l'usure de ces images, comme celle du velours des fauteuils les plus sollicités, ne signifie en aucun cas qu'elles ont perdu leur attrait. Bien au contraire: le respect mêlé de fascination rêveuse que l'enfant éprouve à l'égard des «Guermantes» montre bien que l'image routinisée, dans ce cas, ne correspond en rien à une forme éculée, devenue inopérante. L'extrait qu'on vient de lire permet de mieux comprendre le lien qu'établit Max Weber entre le «stéréotype » et la «magie». Si la magie appelle le stéréotype, aussi bien dans ses rituels (formules, incantations, objets, gestes indéfiniment reproduits à l'identique) que dans ses prolongements artistiques et les formes qu'elle privilégie ${ }^{2}$, l'inverse est également vrai: le stéréotype est

2. «Les innovations symboliques mettent en danger l'efficacité magique des actes ou peuvent même [...] éveiller la colère du dieu ou de l'âme de l'ancêtre. [...] La stéréotypisation religieuse des produits des arts plastiques, qui est le plus ancien mode de constitution d'un style, est conditionnée à la fois directement, par des représentations magiques, et indirectement, par l'apparition d'un mode de production professionnel, qui découle de l'importance magique du produit. [...] L'effet premier, et fondamental, des sphères de représentation "religieuses" sur la conduite de vie et sur l'économie est, de façon générale, un effet de stéréotypisation. Toute modification d'une coutume qui s'opère, d'une façon ou d'une autre, sous la protection de puissances suprasensibles, peut affecter les intérêts d'esprits et de dieux. Aux incertitudes et aux obstacles naturels rencontrés par tout novateur, la religion ajoute ainsi d'autres entraves puissantes: le sacré est ce qui, spécifiquement, ne peut changer». (Weber 2006, 91-93) 
la forme capable de produire l'effet magique, la sacralisation secrète, les attachements immuables qui sont au principe de la révérence à l'égard des traditions. Il existe un lien entre l'autorité traditionnelle, le stéréotype et ses effets magiques. "Le sacré, écrit Max Weber, est ce qui, spécifiquement, ne peut changer »; on peut aussi renverser la proposition et constater l'effet de sacralisation produit par l'immuabilité du stéréotype.

Les images immuables de la tradition tirent leur efficacité, le texte de Proust le suggère, de deux caractéristiques particulières. La première est une certaine polysémie: ce sont des images flottantes (comme les projections de la lanterne magique ou les reflets colorés du vitrail), dont le contenu n'est pas défini de manière restrictive, et qui autorisent un nombre infini de projections individuelles. La seconde est la coupure infranchissable qui sépare les images de la tradition et la "réalité ». Ces images "ne peuvent changer " parce qu'elles ne sont jamais mises à l'épreuve de la réalité, comme s'il s'agissait là de deux sphères fondamentalement incompatibles. Le jeune homme refuse de croire que l'entrée des Enfers puisse, en Grèce, être visitée par des touristes: les images de la tradition sont installées du côté du mythe, si solidement qu'aucune réalité ne peut les faire vaciller. Proust met ainsi le doigt sur le paradoxe le plus remarquable de l'autorité traditionnelle: le type d'autorité le plus établi dans la réalité, le plus "assis », trouve sa source de légitimité la plus efficace dans des images mythiques, et ces images, les plus "routinisées ", sont aussi celles qui exercent non des effets «de routine", mais l'action la plus magique. La progression vers la dématérialisation que nous avons observée dans la série d'images des Guermantes prend dès lors tout son sens: l'honneur de la lignée est sans doute arrimé, aussi puissamment qu'à la pierre de son château de famille, à la silhouette de dame médiévale projetée en couleurs par une lanterne magique, ou à la poésie de son nom. Un tel lien de continuité unit ces images, depuis le château jusqu'à la lanterne et la syllabe, que les plus immatérielles sont encore porteuses de l'autorité de celles qui le sont le moins et qui constituent les insignes de pouvoir les plus tangibles, tandis que, à l'inverse, les images les plus impalpables nimbent aussi les insignes de pouvoir les plus lourds et leur confèrent la délicatesse sans laquelle ils pourraient susciter des réactions de rejet. Ces échanges et circulations entre les images de puissance et les images «éthérées» jouent un rôle fondamental dans la perpétuation des croyances traditionnelles et dans le fonctionnement magique des stéréotypes qui leur sont associés. Il en va de même de la circulation, fluide elle aussi, entre les formes artistiques plus recherchées 
(le château, la tapisserie représentant «le Couronnement d'Esther ») et les formes d'art plus vulgarisées (le vitrail de l'église du village, assez récent et aux teintes un peu fortes, "vert chou» et «bleu prune», et la lanterne magique), entre lesquelles il existe également une continuité — tout comme, en littérature, un même mythe peut inspirer efficacement aussi bien une œuvre savante qu'un roman à grand tirage. Ces continuités n'impliquent pas l'abolition des hiérarchies socialement établies (entre le «savant» et le «populaire», le «matériel » et «l'immatériel») mais permettent à celles-ci d'être «irriguées » de part en part. Dans le texte que nous avons lu, Proust entrecroise ainsi deux hiérarchies qu'il fait progresser en sens inverse, celle qui va du matériel à l'immatériel et celle qui va du grand art aux objets de décoration en série:

Matériel

Architecture dessin

Château contour translucide

vitrail d'Église XIX

vert chou immatériel

reflet qui se déplace

lanterne magique

orangés

art industriel

Grand art

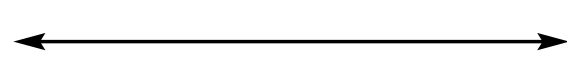

L'impalpable est ici du côté de l'objet fabriqué en grand nombre, tout comme la plus grande qualité de la couleur: la progression vers l'immatériel et vers les «hauteurs» accompagne paradoxalement une perte apparente d' "essence ", puisqu'on va vers des objets de moins en moins uniques. Cette contradiction, subtilement rendue par Proust, décrit le fonctionnement même du stéréotype traditionnel, dans lequel circulent sans relâche la répétition et la réinvention, le quotidien et l'extraquotidien, la puissance et la finesse.

\section{La «déception» charismatique}

Un jour ma mère me dit: «Puisque tu parles toujours de Mme de Guermantes, comme le docteur Percepied l'a très bien soignée il y a quatre ans, elle doit venir à Combray pour assister au mariage de sa fille. Tu pourras l'apercevoir à la cérémonie ». C'était du reste par le docteur Percepied que j'avais le plus entendu parler de Mme de Guermantes, et il nous avait même montré le numéro d'une revue illustrée où elle était représentée dans le costume qu'elle portait à un bal travesti chez la Princesse de Léon. 
Tout d'un coup pendant la messe de mariage, un mouvement que fit le suisse en se déplaçant me permit de voir assise dans une chapelle une dame blonde avec un grand nez, des yeux bleus et perçants, une cravate bouffante en soie mauve, lisse, neuve et brillante, et un petit bouton au coin du nez. Et parce que dans la surface de son visage rouge, comme si elle eût eu très chaud, je distinguais, diluées et à peine perceptibles, des parcelles d'analogie avec le portrait qu'on m'avait montré, parce que surtout les traits particuliers que je relevais en elle, si j'essayais de les énoncer, se formulaient précisément dans les mêmes termes: un grand nez, des yeux bleus, dont s'était servi le docteur Percepied quand il avait décrit devant moi la Duchesse de Guermantes; or la chapelle où elle suivait la messe était celle de Gilbert le Mauvais, sous les plates tombes de laquelle, dorées et distendues comme des alvéoles de miel, reposaient les anciens comtes de Brabant, et que je me rappelais être à ce qu'on m'avait dit réservée à la famille de Guermantes quand quelqu'un de ses membres venait pour une cérémonie à Combray; il ne pouvait vraisemblablement y avoir qu'une seule femme ressemblant au portrait de Mme de Guermantes, qui fût ce jour-là, jour où elle devait justement venir, dans cette chapelle: c'était elle! Ma déception était grande. Elle provenait de ce que je n'avais pas pris garde quand je pensais à Mme de Guermantes, que je me la représentais avec les couleurs d'une tapisserie ou d'un vitrail, dans un autre siècle, d'une autre matière que le reste des personnes vivantes. Jamais je ne m'étais avisé qu'elle pouvait avoir une figure rouge, une cravate mauve comme Mme Sazerat, et l'ovale de ses joues me fit tellement souvenir de personnes que j'avais vues à la maison que le soupçon m'effleura, pour se dissiper d'ailleurs aussitôt après, que cette dame en son principe générateur, en toutes ses molécules, n'était peut-être pas substantiellement la Duchesse de Guermantes, mais que son corps, ignorant du nom qu'on lui appliquait, appartenait à un certain type féminin, qui comprenait aussi des femmes de médecins et des commerçants. "C'est cela, ce n'est que cela, Mme de Guermantes! », disait la mine attentive et étonnée avec laquelle je contemplais cette image qui naturellement n'avait aucun rapport avec celles qui sous le même nom de Mme de Guermantes étaient apparues tant de fois dans mes songes, puisque, elle, elle n'avait pas été comme les autres arbitrairement formée par moi, mais qu'elle m'avait sauté aux yeux pour la première fois il y a un moment seulement, dans l'église; qui n'était pas de la même nature, n'était pas colorable à volonté comme celles qui se laissaient imbiber de la teinte orangée d'une syllabe, mais était si réelle que tout, jusqu'à ce petit bouton qui s'enflammait au coin du nez, certifiait son assujettissement aux lois de la vie, comme dans une apothéose de théâtre, un plissement de la robe de la fée, un tremblement de son petit doigt, dénoncent la présence matérielle d'une actrice vivante, là où nous étions incertains si nous n'avions pas devant les yeux une simple projection lumineuse. 
Mais en même temps, sur cette image que le nez proéminent, les yeux perçants, épinglaient dans ma vision (peut-être parce que c'était eux qui l'avaient d'abord atteinte, qui y avaient fait la première encoche, au moment où je n'avais pas encore le temps de songer que la femme qui apparaissait devant moi pouvait être Mme de Guermantes), sur cette image toute récente, inchangeable, j'essayais d'appliquer l'idée: "C'est Mme de Guermantes » sans parvenir qu'à la faire manœuvrer en face de l'image, comme deux disques séparés par un intervalle. Mais cette Mme de Guermantes à laquelle j'avais si souvent rêvé, maintenant que je voyais qu'elle existait effectivement en dehors de moi, en prit plus de puissance encore sur mon imagination qui, un moment paralysée au contact d'une réalité si différente de ce qu'elle attendait, se mit à réagir et à me dire: "Glorieux dès avant Charlemagne, les Guermantes avaient le droit de vie et de mort sur leurs vassaux; la Duchesse de Guermantes descend de Geneviève de Brabant. Elle ne connaît, ni consentirait à connaître aucune des personnes qui sont ici ».

$\mathrm{Et}$ - ô merveilleuse indépendance des regards humains, retenus au visage par une corde si lâche, si longue, si extensible qu'ils peuvent se promener seuls loin de lui - pendant que Mme de Guermantes était assise dans la chapelle au-dessus des tombes de ses morts, ses regards flânaient çà et là, montaient le long des piliers, s'arrêtaient même sur moi, comme un rayon de soleil errant sur la nef, mais un rayon de soleil qui, au moment où je reçus sa caresse, me sembla conscient. Quant à Mme de Guermantes elle-même, comme elle restait immobile, assise comme une mère qui semble ne pas voir les audaces espiègles et les entreprises indiscrètes de ses enfants qui jouent et interpellent des personnes qu'elle ne connaît pas, il me fut impossible de savoir si elle approuvait ou blâmait dans le désœuvrement de son âme, le vagabondage de ses regards.

Je trouvais important qu'elle ne partît pas avant que j'eusse pu la regarder suffisamment, car je me rappelais que depuis des années je considérais sa vue comme éminemment désirable, et je ne détachais pas mes yeux d'elle, comme si chacun de mes regards eût pu matériellement emporter et mettre en réserve en moi le souvenir du nez proéminent, des joues rouges, de toutes ces particularités qui me semblaient autant de renseignements précieux, authentiques et singuliers sur son visage. Maintenant que me le faisaient trouver beau toutes les pensées que j'y rapportais - et peut-être surtout, forme de l'instinct de conservation des meilleures parties de nous-mêmes, ce désir qu'on a toujours de ne pas avoir été déçu — la replaçant (puisque c'était une seule personne qu'elle et cette Duchesse de Guermantes que j'avais évoquée jusque-là) hors du reste de l'humanité dans laquelle la vue pure et simple de son corps me l'avait fait un instant confondre, je m'irritais en entendant dire autour de moi: «Elle est mieux que Mme Sazerat, que Mlle Vinteuil», comme si elle leur 
eût été comparable. Et mes regards s'arrêtant à ses cheveux blonds, à ses yeux bleus, à l'attache de son cou et omettant les traits qui eussent pu me rappeler d'autres visages, je m'écriais devant ce croquis volontairement incomplet: "Qu'elle est belle! Quelle noblesse! Comme c'est bien une fière Guermantes, la descendante de Geneviève de Brabant, que j'ai devant moi! ». (Proust 1987, 287-290)

Ce deuxième passage est construit comme le contrepoint du premier: alors que la description des images mythiques des Guermantes représentait une progression vers l'immatériel, le récit de la rencontre avec la duchesse confronte le jeune héros avec les aspérités du concret. Tandis que le premier mouvement l'emportait vers des mondes rêvés, dans lesquels son imagination trouvait à s'épanouir, le contact avec cette réalité le heurte et le plonge dans «l'étonnement». Le début du texte est cette fois centré autour de la phrase la plus brève, qui résume le sentiment dominant: «Ma déception était grande». Déçu, le jeune homme ne l'est pas seulement dans ses attentes, parce que la dame qu'il a sous les yeux est différente de celle qu'il avait imaginée ( C'est cela [...]!»); il l'est aussi et surtout parce qu'elle est moins belle et moins brillante («ce n'est que cela [...]! »). La duchesse réelle partage des propriétés avec d'autres femmes, elle appartient même à un "type ", là où son image mythique lui conférait une singularité incomparable. L'éclat délicieux de la généalogie ( «dorées et distendues comme des alvéoles de miel») est démenti par un vocabulaire de couleurs électriques et vulgaires ( «des yeux bleus et perçants, une cravate bouffante en soie mauve, lisse, neuve et brillante», "une figure rouge ») et le jeune homme cherche en vain les signes de distinction attendus, dans le vêtement comme dans le visage.

L'expérience charismatique n'est pas l'antithèse d'une telle «déception ». Elle en est, en toute rigueur, le produit. Elle est un phénomène second qui naît d'un moment de négativité initiale dans lequel les croyances traditionnelles se trouvent suspendues. La foi charismatique est une croyance d'un type nouveau qui vient remplacer l'ancienne; elle n'annule pas l'allégeance à l'autorité traditionnelle mais la met à l'épreuve, la perturbe, la place dans un rapport de tension avec le réel. La croyance charismatique surgit dans un entre-deux, lorsque la positivité de la tradition, son inertie, est bousculée par la négativité du réel qui balaie les images mythiques. Le charisme, comme le dit Max Weber, est une puissance «révolutionnaire» (Weber 1972, 657; Kalinowski 2005, 126 sq.), il comporte d'abord cette dimension de rupture, de décalage soudain entre deux représentations, l'image traditionnelle et la vision réelle. On a devant soi «deux disques 
séparés par un intervalle » et on ne parvient plus à les faire coïncider. Cette formule de Proust possède une grande pertinence et décrit précisément le lieu d'émergence de la croyance charismatique, qui est un espace instable. Voilà pourquoi l'exclamation finale du jeune homme, convaincu en dernière instance de la beauté et de la noblesse de madame de Guermantes, n'est pas la conséquence logique du point de vue qui était à l'origine le sien lorsque l'évocation du château ou le reflet de la lanterne magique le faisaient rêver. Entre le début et la fin du texte, où tout semble «rentré dans l'ordre ", l'ordre a justement été bouleversé, et ce n'est plus la déférence à l'égard de la tradition qui commande, à elle seule, le jugement ultime de Marcel. La «déception", si elle semble ne durer qu'un temps, ne se limite cependant pas à un doute momentané: c'est une perte fondamentale et irrémédiable par rapport à la naïveté de la croyance première, qui n'est pas restaurée à la fin. Bien qu'il évoque le «désir qu'on a toujours de ne pas avoir été déçu» et voie en lui une "forme de l'instinct de conservation », le narrateur ne méconnaît pas la réalité de cette transformation et n'ignore pas que le jeune héros est passé d'un registre de croyance à un autre.

L'autorité traditionnelle donne toute sa mesure dans une distance indéfiniment préservée, celle du mythe, l'écart qui sépare le château du village, ou encore la hauteur inaccessible d'une tapisserie, d'un vitrail ou d'un reflet de lanterne magique; de même, la dimension répétitive et stéréotypée des rites traditionnels les soustrait au temps ordinaire et aux modifications continuelles qui affectent la vie quotidienne. Tout à l'inverse, l'autorité charismatique ne s'affirme pas ailleurs que dans l'ici et maintenant. Elle naît dans l'actualité d'une perception qui ne croit que ce qu'elle voit. N'est charismatique que ce qu'on a "sous les yeux" : le charisme est une performance, comme la «virtuosité » (reconnue en acte et jamais comme une simple potentialité). L'autorité traditionnelle n'a pas nécessairement besoin de s'incarner dans un corps réel et de se placer, littéralement, "sous les yeux" de ses adeptes; ceux-ci peuvent se contenter de prendre pour référents des images, des «chromos" plus ou moins éthérés. Au contraire, le pouvoir charismatique n'est jamais attribué qu'à un corps de chair qui donne à voir son épaisseur, sa peau, ses couleurs, ses gestes, le rythme de ses mouvements, et, le cas échéant, fait entendre sa voix.

Pourtant, en dépit de son caractère de performance, le charisme se distingue de la virtuosité au sens où il n'exige pas nécessairement la démonstration d'une habileté particulière. Ainsi que le récit de la rencontre de Marcel et de la duchesse le souligne avec insistance, la première et la principale 
performance du charismatique, c'est d'être là, avec les propriétés qui le caractérisent à un moment donné, et non d'accomplir tel ou tel acte. Si ce qui émane du corps du charismatique est généralement le produit d'un long apprentissage, comme la technique du virtuose, il n'a pas, à la différence de ce dernier, à donner la confirmation effective d'un savoir-faire ou d'un art. À la limite, la croyance charismatique peut naître, comme dans la scène de l'église, de la simple vision d'un personnage assis, immobile et muet. Ce qui est réellement agissant, notre texte l'affirme exemplairement, c'est un corps. La croyance charismatique se confond, initialement, avec la perception d'un corps. Celle-ci se focalise dans un premier temps sur un élément saillant, comme le bouton sur le nez ou les rougeurs de la peau. Mais le regard devine bientôt que l'essentiel ne se joue peut-être pas au niveau des détails les plus explicites de la surface. Le corps acquiert une épaisseur qui ne tarde guère à rendre caducs les jugements péremptoires des premiers instants. Dans le portrait de la duchesse, le passage du détail isolé à la personnification des yeux (dont le "vagabondage» évoque pour Marcel «une mère " assise au milieu de ses enfants et feignant d'ignorer leurs "audaces espiègles ") traduit ce gain de densité: le corps cesse d'être une simple somme de caractéristiques portées comme des accessoires ( une dame blonde avec un grand nez, des yeux bleus et perçants, une cravate bouffante en soie mauve, lisse, neuve et brillante, et un petit bouton au coin du nez») pour devenir une personnalité, un système vivant dont la clé se dérobe tout en s'affirmant de manière pénétrante pour celui qui le regarde.

Le mouvement qui déploie ainsi la perception physique trouve son pendant dans celui qui se déplace du visage, la partie qui constitue son premier point focal, au reste du corps. Le visage semble concentrer les enjeux les plus sensibles du physique du charismatique, et c'est d'abord lui qui retient l'attention, mais il n'est pas le lieu exclusif de l'expressivité. L'importance qui lui est accordée tient davantage au fait qu'il représente en quelque sorte un résumé du corps tout entier, comme, dans la médecine chinoise, le lobe de l'oreille ou la plante des pieds sont reliés à l'ensemble des organes humains. Le visage microcosme du corps porte sur lui des marques auxquelles correspondent, à une échelle plus étendue, certains gestes, certaines lignes de mouvements ou encore certains vêtements et singularités de la tenue. En fin de compte, ce n'est pas seulement le visage mais chaque partie, si elle est finement observée, qui révèle la texture particulière de l'ensemble.

Si l'objet de la croyance charismatique est une propriété qui se fait sentir à même le corps, la rencontre dans l'église de Combray invite à en préciser 
la teneur. Le texte de Proust, comme bien d'autres scènes de la Recherche, suggère une hypothèse sur la genèse du charisme: celui-ci serait, spécifiquement, l'effet produit par une tension entre des caractéristiques d'abord perçues comme contradictoires. La perception d'une telle contradiction, profondément déconcertante, serait à l'origine du choc charismatique. Le charisme ne résiderait pas de prime abord dans le contenu positif, grâce, talent, charme, don, valorisé par ses adeptes, mais plutôt dans une structure conflictuelle opposant certaines propriétés spécifiques de son porteur; c'est l'antagonisme de ces propriétés, et non leur nature particulière, qui importerait avant tout. L'extrait que nous venons de lire est même plus explicite encore. Il identifie le charisme comme la combinaison d'une autorité de type traditionnel et de composantes qui ne correspondent pas aux attributs de l'autorité traditionnelle. Dans le cas (paradigmatique) de la duchesse de Guermantes, la contradiction qui «saute aux yeux» du jeune homme est la conjonction du prestige familial et de la simplicité de la mise. Elle est ici d'autant plus patente, dans un effet de mise en scène démonstratif, que «Mme de Guermantes était assise dans la chapelle au-dessus des tombes de ses morts". Le charisme s'expose dans la réunion contrastée d'un certain corps vivant et d'un certain héritage. Quelque chose, dans ce rapprochement, ne va pas de soi.

Avant de la rencontrer, Marcel était incapable d'imaginer son héroïne autrement que dans des atours redoublant sa qualité sociale, des costumes précieux qui auraient matérialisé ce qui la séparait des autres femmes et, dans leur raffinement, l'auraient rendue indéchiffrable. Il la voyait tout à fait, comme dans le magazine apporté par le docteur Percepied, dans un «bal travesti chez la Princesse de Léon ». Les déguisements et les costumes étaient pour lui les attributs naturels de cette femme inaccessible. Le désarroi dans lequel le plonge, par suite, la vision de sa "peau ", sujette aux «rougeurs» comme une vraie peau, est immense. À proprement parler, la contradiction qui le frappe ainsi n'est pas celle qui opposerait le «rêve ", ou le «mythe », et la «réalité », par exemple l'image rêvée d'un visage exquis et les imperfections réelles de son teint. Ce n'est pas le manque de beauté de madame de Guermantes qui sidère Marcel, mais d'abord le fait qu'elle soit «vivante» et assujettie «aux lois de la vie»: le bouton sur le nez ne connote pas la laideur mais bien plutôt l'existence. La perception d'une particularité physique traduit seulement la stupeur de voir prendre corps ce qui était censé appartenir à un autre ordre que celui du corps. L'ébranlement charismatique naît de cet étonnement. Dans cette logique, la 
figure de l'acteur ou de "l'actrice» représente un modèle par excellence de figure charismatique, dans la mesure où elle opère directement la réunion d'un «rôle» et d'un corps vivant: le spectateur de théâtre entre dans un régime de croyance nouveau lorsqu'il réalise qu'il a devant lui, non plus seulement une fée, mais une fée dont le petit doigt tremble.

Dans un autre volume de la Recherche, le récit de la première confrontation de Marcel avec la grande actrice de son temps, la Berma, prolonge les observations qui sont faites dans notre texte au sujet de la croyance charismatique. Après avoir longtemps insisté auprès de ses parents pour être autorisé à aller voir jouer la comédienne dont la renommée le fascine, le jeune homme assiste finalement à une représentation de Phèdre dans laquelle celle-ci tient le rôle éponyme. Comme dans l'église de Combray, il vit sa première rencontre avec cette femme longtemps rêvée comme une déception. Sidéré de découvrir en elle tout autre chose que ce qu'il avait imaginé, rebuté par une diction monotone qui lui semble au premier abord moins soignée que celle "des élèves de lycée », Marcel est contraint d'abandonner sans délai la croyance «traditionnelle» qui était à l'origine la sienne, et que les applaudissements nourris du public ne suffisent pas à raviver. Là encore, le passage à cet autre registre d'adhésion qu'est la croyance charismatique ne se fonde pas sur la perpétuation de l'allégeance traditionnelle; elle en suppose au contraire la suspension. C'est la tension entre la connaissance du prestige artistique de la Berma et la perception de cette femme comme une présence vivante qui provoque le basculement dans le régime charismatique.

L'apparition réelle de la comédienne instaure tout à la fois une brusque limitation des possibles, que le jeune homme ressent en se désolant de «l'uniformité » de sa voix par rapport à celles qu'il lui attribuait auparavant en imagination ( "Elle passa au rabot d'une mélopée uniforme toute la tirade $[. .] »$.$) , et l'ouverture de l'accès à une personnalité qu'il est incapable$ de cerner d'emblée mais qui éveille fortement sa curiosité. Dans l'expérience charismatique, la perception du corps de l'autre dans sa présence impose un recadrage brutal des attentes de l'adepte: elles se trouvent immédiatement restreintes et, en même temps, intensifiées dans leur périmètre nouveau. La croyance charismatique se distingue ainsi de la croyance traditionnelle par une moindre extension et une plus forte intensité. La première tend à l'individualisation là où la seconde maintient la possibilité d'une généralisation. L'autorité charismatique est individualisée non seulement parce qu'elle s'affirme à travers la présence physique particulière 
du "charismatique », mais aussi parce que celle-ci ne peut s'affirmer qu'auprès d'un nombre limité d'individus dont les propriétés sont en affinité avec les siennes. Cette forme d'autorité individualise à la fois son porteur et ses adeptes.

\section{Conclusion}

Les observations livrées par Proust au sujet de la genèse de la croyance charismatique, dont le récit de la rencontre de Marcel avec la duchesse de Guermantes fournit un exemple significatif, rejoignent et précisent, sur le terrain de l'écriture littéraire, les analyses du phénomène charismatique développées par Max Weber avec les outils de la sociologie.

En premier lieu, le texte soulève le problème des rapports entretenus par deux modes d'autorité concurrents, le mode traditionnel et le mode charismatique, tout en mettant l'accent sur la proximité qui les unit. Dans sa fameuse partition entre les "trois types purs d'autorité légitime», "traditionnelle ", "charismatique» et "rationnelle bureaucratique ", Max Weber opère lui aussi un rapprochement entre les deux premières: même si l'autorité charismatique partage avec la troisième le moment "rationnel » du refus de l'allégeance à la tradition pour la tradition, la croyance charismatique ne prend pas pour objet des individus dépourvus de tout «capital» traditionnel; au contraire, elle est exacerbée par la conjonction, chez le charismatique, de propriétés traditionnelles et de caractéristiques «antitraditionnelles ». Dans la sociologie wébérienne, la notion apparemment contradictoire de «charisme de fonction» pointe l'existence d'une zone d'affinité et de tension fortes entre l'autorité traditionnelle et l'autorité charismatique. En toute rigueur, le charisme n'est jamais «de fonction", dérivé d'une autorité traditionnelle, mais, d'un certain point de vue, tout charisme est «de fonction», appuyé sur la tension avec une propriété traditionnelle ${ }^{3}$.

En second lieu, la description de la première rencontre avec la duchesse développe un constat récurrent dans l'univers proustien comme dans la sociologie wébérienne: la croyance charismatique s'élabore en présence d'un corps physique. Ainsi que le texte le souligne avec insistance, ce n'est pas la qualité esthétique du corps, positive ou négative, qui est au principe de cette croyance, mais plutôt l'effet exercé sur le futur adepte par le corps

3. Cette analyse est développée dans le premier chapitre («Charisme et charisme de fonction ") d'un ouvrage en préparation intitulé Le charisme. Autour de Max Weber. 
dans ce qu'il a de plus visible, son exhibition, sa présence perceptible, et, en même temps, de plus insaisissable, sa façon de condenser des propriétés qui demeurent implicites. Le corps charismatique radicalise la contradiction de tout corps: il montre et cache à la fois, il offre à la vue tout en soustrayant au regard, il est la cristallisation d'une histoire qu'il exhibe et renvoie dans le même instant à l'état tacite. Ce corps est de part en part social, il porte les insignes d'une position, d'un parcours, depuis les plus manifestes jusqu'aux plus discrets; mais il s'emploie simultanément - ce qui ne revient pas au même — à «naturaliser » ces propriétés sociales, c'est-à-dire à faire oublier qu'elles ont une histoire et à les faire percevoir comme naturelles. Le texte de Proust explore avec une grande acuité le désarroi du jeune héros devant ce corps qui déjoue ses premières tentatives de classement: tel un sociologue, il cherche d'abord à l'objectiver, à en énumérer les propriétés, les "avoirs ", cravate mauve et autres attributs; mais, échouant dans cette entreprise, il abdique devant la croyance charismatique et s'en remet à sa foi nouvelle dans «l'être» de la duchesse. Le charisme pourrait ainsi être défini comme un prestige "fait corps", mais pas au sens où il prolongerait dans un corps vivant des propriétés sociales aisément identifiables: si l'on peut dire que le charisme est "incarné ", c'est d'abord au sens où il se fond parfaitement avec le corps, et présente à tel point les apparences de la naturalité qu'on est porté à oublier qu'il est un prestige et pas seulement un corps. Il se présente fondamentalement comme un corps, et la contradiction entre ce corps et les propriétés "prestigieuses " de l'individu est le critère décisif de l'efficace du phénomène charismatique. Autour de ce corps, le jeu charismatique peut battre son plein: il implique à la fois une relation hiérarchisée et strictement dissymétrique (Marcel garde les yeux fixés sur la duchesse dont le regard, central, erre sans objet précis) et un engagement actif de celui vers lequel convergent tous les points de vue: la duchesse doit être présente physiquement et "donner de sa personne" pour réactiver la croyance charismatique, sa venue dans la chapelle des Guermantes n'a rien de fortuit. Elle doit s'y " offrir aux regards » parce que ces derniers lui sont nécessaires. Ces observations proustiennes sur le corps charismatique rejoignent directement ce que Max Weber, de son côté, avait résumé dans la notion de "charisme héréditaire" (voir Kalinowski 2005, 134-142): la tension entre les propriétés héritées et la nécessité de leur réactualisation à chaque fois recommencée dans le corps physique d'un individu, qui doit tout à la fois les rendre sensibles et les faire oublier. 


\section{Références}

Balzac, H. de (1978), "Physiologie gastronomique», dans Théorie de la démarche et autres textes, Paris, Pandora, p. 145-155.

Haley, P. (1980), «Rudolph Sohm on Charisma », Journal of Religion, 60/2, p. 185-197.

Holl, K. (1898), Enthusiasmus und Bußgewalt beim griechischen Mönchtum. Eine Studie zu Symeon, dem neuen Theologen, Leipzig, J. C. Hinrich.

KALINOwSKI, I. (2005), Leçons wébériennes sur la science et la propagande, Marseille, Agone.

Proust, M. (1987), "Combray», dans Du côté de chez Swann, Paris, Flammarion.

RIEFF, P. (2007), Charisma. The Gift of Grace, and How it Has Been Taken Away from Us, New York, Pantheon Books.

SMITH, D. N. (1998), «Faith, Reason, and Charisma. Rudolf Sohm, Max Weber, and the Theology of Grace ", Sociological Inquiry, 68/1, p. 32-60.

SoHM, R. (1912), Wesen und Ursprung des Katholizismus, Leipzig/Berlin, Teubner.

Weber, M. (19725), Wirtschaft und Gesellschaft, Tübingen, Mohr. (2000), L'éthique protestante et l'esprit du capitalisme, trad. par I. Kalinowski, Paris, Flammarion.

(2003), Hindouisme et bouddhisme, trad. par I. Kalinowski et R. Lardinois, Paris, Flammarion.

(2006), Sociologie de la religion, trad. par I. Kalinowski, Paris, Flammarion.

(2010), Le judaïsme antique, trad. par I. Kalinowski, avec la collaboration de C. Joseph et B. Lévy, Paris, Flammarion. 


\section{Résumé}

Cet article propose une lecture sociologique du passage de $\grave{A}$ la recherche $d u$ temps perdu où le jeune Marcel rencontre la duchesse de Guermantes et réaffirme, après un premier moment de "déception", sa croyance dans le "charisme» de cette dame. Fondé sur la théorie du charisme de Max Weber, ce commentaire cherche à prolonger cette dernière en insistant sur deux dimensions mises au jour par Proust: la différence entre croyance charismatique et croyance "traditionnelle », d'une part, et, de l'autre, le rôle de la perception du corps dans la genèse de l'enchantement charismatique.

\section{Abstract}

This article presents a sociological reading of an excerpt from À la recherche du temps perdu where young Marcel meets the Duchesse de Guermantes. After a first moment of "disappointment ", Marcel reasserts his belief in the "charisma" of the lady. Based on Max Weber's charisma theory, this commentary develops the latter and insists on two dimensions brought out by Proust: on the first hand the difference between charismatic belief and "traditional» belief, and, on the second hand, the role that the perception of the body plays in the genesis of the charismatic enchantment. 